\title{
Optimal LTE-Protected LPDA Design for DVB-T Reception Using Particle Swarm Optimization with Velocity Mutation
}

\author{
Zaharias D. Zaharis, Senior, Member, IEEE, Ioannis P. Gravas, Student, Member, IEEE, Pavlos I. \\ Lazaridis, Senior, Member, IEEE, Ian A. Glover, Member, IEEE, Christos S. Antonopoulos, Senior, \\ Member, IEEE, and Thomas D. Xenos
}

\begin{abstract}
A near-optimal design of a log-periodic dipole array (LPDA), suitable for DVB-T reception (470-790 MHz), is presented. The LPDA is required to provide low standing wave ratio as well as high-gain radiation pattern with sufficient gain flatness over the entire passband, and concurrently achieve low gain for frequencies above $800 \mathrm{MHz}$ to reject LTE800 signals and thus improve the reception quality in the DVB-T band. All the above requirements are better satisfied by applying a novel particle swarm optimization (PSO) variant, called PSO with velocity mutation (PSOvm). PSOvm induces mutation on the velocities of those particles, which are unable to improve their fitness. As shown in this paper, PSOvm comes closer to the above requirements compared to four well-known optimization methods and outperforms the traditional LPDA design method proposed by Carrel. The LPDA geometry chosen for optimization is not the conventional one and therefore the dipoles are not considered to be included inside a specified angle as proposed by Carrel. Thus, the dipole lengths and distances as well as the boom dimensions are independently optimized. The PSOvm-based LPDA sufficiently meets all the above requirements and thus is suitable for DVB-T reception without the use of an external LTE-band rejection filter.
\end{abstract}

Index Terms-Antenna optimization, DVB-T, log-periodic antennas, log-periodic dipole arrays (LPDAs), LTE, particle swarm optimization

\section{INTRODUCTION}

$\mathrm{L}$ OG periodic dipole arrays (LPDAs) are special antenna $\boldsymbol{J}_{\text {structures composed of dipoles of different length. At a }}$ specified frequency, the current distribution has important values only along some dipoles with proper length, which means that only these dipoles radiate at the specified frequency. The result is a wideband behavior, which depends

Zaharias D. Zaharis, Ioannis P. Gravas, Christos S. Antonopoulos, and Thomas D. Xenos are with the Department of Electrical and Computer Engineering, Aristotle University of Thessaloniki, Thessaloniki 54124, Greece (e-mail: zaharis@auth.gr; igravas@auth.gr; chanto@auth.gr; tdxenos@auth.gr).

Pavlos I. Lazaridis and Ian A. Glover are with the Department of Engineering and Technology, University of Huddersfield, Huddersfield HD1 3DH, United Kingdom (e-mail: p.lazaridis@hud.ac.uk; i.a.glover@hud.ac.uk). on the number of dipoles, as well as on their lengths, distances and radii [1]. A parameter that highlights this behavior is the gain flatness $(G F)$, which is defined as the difference between the maximum value and the minimum value of the forward gain, $F G_{\max }$ and $F G_{\min }$ respectively, found over the entire operating band (i.e., $G F=F G_{\max }-F G_{\text {min }}$ ). Therefore, LPDAs are very useful in applications where a wideband behavior is required, such as TV and FM-radio reception, wideband precision measurements, electromagnetic compatibility (EMC) measurements and spectrum surveillance.

Typically, the LPDAs have lower forward gain $(F G)$ in comparison to Yagi-Uda antennas, [1], but with smaller gain variations over the entire frequency range of operation. On the contrary, higher $F G$ is achieved by Yagi-Uda antennas over a much narrower bandwidth and the gain variations over the entire operating band are higher. Due to their good (i.e., low) $G F$, LPDAs are more desirable than Yagi-Uda antennas in the above-mentioned applications. The low $F G$ of LPDAs can easily be improved by using arrays of LPDAs, and thus high $F G$ and good $G F$ can concurrently be obtained.

Recently, the restriction of DVB-T in the range 470-790 $\mathrm{MHz}$ together with its proximity to the frequency range of LTE800 have made the rejection of LTE signals become essential for the quality of DVB-T reception. For DVB-T reception, LTE signals are considered as interference and thus they must be eliminated either by using a properly designed external filter connected to a conventional receiving antenna or alternatively by just employing a properly designed $L T E$ protected antenna, which is more cost-effective than the combination of a conventional antenna and an external filter. To the best of our knowledge, no research has been conducted on such antennas so far and especially on LTE-protected LPDAs. An effort to optimize an LTE-protected LPDA under several requirements inside the DVB-T and LTE 800 bands by applying evolutionary optimization methods is carried out in this paper.

The first complete LPDA design procedure was proposed by Carrel in [2] and corrected by Butson and Thompson in [3]. This procedure is a practical design method, where all the dipoles are considered inside a specified angle. According to this method, the calculation of the dipole lengths, distances 
and radii is based on the values of two design parameters, defined as scale factor $\tau$ and relative spacing $\sigma$. These parameters are estimated from the constant directivity contour curves of the well-known Carrel's graph, [1]-[3]. Since then, many software packages based on the above procedure have been released in order to make the LPDA design easier and faster, [4], [5].

However, the above procedure is an approximate design method and thus it cannot be used to accurately control the radiation characteristics for every frequency over the entire operating band. A full wave analysis method would be more appropriate for LPDAs, because it provides the ability to precisely calculate all the radiation characteristics of an antenna over a wide band. Finally, Carrel's method cannot provide signal rejection outside the required operating band, because the objective of this method is to synthesize an antenna geometry with smooth variation of radiation characteristics inside a specified band without being concerned about the variation of these characteristics outside this band. Therefore, an optimization method in conjunction with a full wave analysis method would constitute a powerful design technique suitable for LPDA design under multiple requirements including signal rejection outside the operating band.

Such an optimization method proposed in the present study is a novel variant of the well-known Particle Swarm Optimization (PSO) method [6]-[35], called PSO with velocity mutation (PSOvm). PSOvm employs a mutation mechanism in order to cause perturbation on the velocities of those particles, which are not able to improve their fitness. This mechanism helps PSOvm to achieve better fitness values compared to well-known methods. As shown in [36], in the same computational time (i.e., for the same number of fitness function evaluations), the mean value and the standard deviation of the final fitness achieved by PSOvm are better than the respective values achieved by the conventional Constriction Coefficient PSO (CCPSO) [32], a Differential Evolution (DE) algorithm based on the popular DE/rand/1/bin strategy [37], [38] and the conventional Invasive Weed Optimization (IWO) method [39]-[41] for the majority of the test functions studied in [36].

In the present work, PSOvm, CCPSO [32], DE (based on DE/rand/1/bin strategy) [37], [38], IWO [39]-[41], and a simple genetic algorithm (GA) [42] are comparatively applied to design LPDAs that concurrently satisfy requirements for low standing wave ratio ( $S W R$ ), high $F G$ and low $G F$ over the DVB-T band as well as an additional requirement for low $F G$ above $800 \mathrm{MHz}$ to ensure rejection of LTE800 signals and thus improve the quality of signal reception in the DVB-T band. To have precise calculations of the antenna radiation characteristics, each one of the above five methods is combined with a full wave analysis software. This software has been chosen here to be the CST Microwave Studio (CST MWS) [43]. Actually, CST MWS performs full-wave timedomain analysis in order to calculate the radiation characteristics of the antenna and thus evaluate the fitness function, whenever a fitness evaluation is required by any of the above five methods.

The challenge and also novelty in the present work is that three requirements concerning $S W R, F G$ and $G F$ must concurrently be satisfied inside the passband (DVB-T), while a fourth requirement concerning low $F G$ must simultaneously be satisfied in the stopband (LTE800). Furthermore, the proposed LPDA geometry used by the above five optimization methods is not the conventional (Carrel's) geometry but an arbitrary one, where the dipole lengths and distances as well as the boom dimensions are independently optimized. Finally, the intention of this paper is to show that the proposed method (PSOvm) can come closer to all requirements (defined below) compared to CCPSO, DE, IWO and GA, and is also capable of producing an LPDA with better behavior than that of a conventional (Carrel's) LPDA with the same number of dipoles and the same total length.

\section{PRoblem Formulation AND PRoposed Geometry}

PSOvm, CCPSO, DE, IWO and GA are applied to optimize under several requirements a realistic LPDA geometry modeled in CST MWS and composed of 10 wire dipoles $(M=10)$ in order to be used for signal reception in the DVB-

T UHF band (470-790 MHz) and concurrently reject LTE800 signals (i.e., signals above $791 \mathrm{MHz}$ ). Since the gap between the two bands (DVB-T and LTE800) is only $1 \mathrm{MHz}$ and it is practically hard to satisfy all the requirements given below, we have decided to use a wider transition band, which is henceforth defined to be the range $780-800 \mathrm{MHz}$. In particular, for every frequency in the range $470-780 \mathrm{MHz}$ (passband), the LPDA is required to achieve (i) $S W R \leq 1.9$ (impedance matching condition), (ii) a radiation pattern with the highest possible $F G$, and (iii) $G F \leq 2.5 \mathrm{~dB}$. An additional (fourth) requirement is set for the stopband to ensure rejection of LTE800 signals (and thus improve the quality of signal reception in the DVB-T band): $F G \leq 0 \mathrm{dBi}$ for every frequency in the range $800-900 \mathrm{MHz}$ (stopband), which means that $F G$ is required to exhibit a rapid decrease inside the 20 $\mathrm{MHz}$ width of the transition band. It must be noted that, although the desired $S W R$ is usually 2:1 for signal reception, the more strict value of 1.9:1 has been chosen to ensure that even in practice the antenna will satisfy the impedance matching condition for every frequency inside the passband.

To satisfy the above four requirements, we propose an arbitrary LPDA geometry (see Fig. 1), where the dipole lengths and distances as well as the boom dimensions are independently optimized. Therefore, the dipoles are not considered to be included inside a specified angular sector as is the case in Carrel's geometry [1]. It is believed that this type of geometry in combination with an evolutionary optimization method induces a much greater design freedom in comparison to Carrel's method, and therefore the concurrent satisfaction of the four requirements specified above may be achieved more easily. Moreover, the front ( $M$-th) dipole must be larger than the previous $((M-1)$-th) one in order to start acting like a 
reflector at the upper limit of the passband and thus force $F G$ to rapidly decrease inside the transition band.

To facilitate the construction of the dipoles in practice, all the dipole radii $r_{m}(m=1, \ldots, M)$ are considered fixed and equal to the typical value of $2 \mathrm{~mm}$. So, the optimization variables are the dipole lengths $L_{m}(m=1, \ldots, M)$, the distances between adjacent dipoles $S_{m}(m=1, \ldots, M-1)$, the distance $S_{M}$ between the front ( $M$-th) dipole and the feeding point of the antenna, the thickness $d_{y}$ of each rod of the boom along y-direction, and finally the spacing $s_{z}$ between the closest surfaces of the rods (Fig. 1). The thickness $d_{z}$ of each rod of the boom along z-direction is considered fixed and equal to the dipole diameter, i.e., $4 \mathrm{~mm}$. This value is practically the lowest possible value that provides the ability to attach the dipoles to the rods of the boom, and it was chosen after several trials which revealed that a decrease in $d_{z}$ improves the antenna radiation characteristics. Finally, each one of the above five optimization methods has to find proper values for 22 optimization variables $(D=2 M+2=22)$ in total.

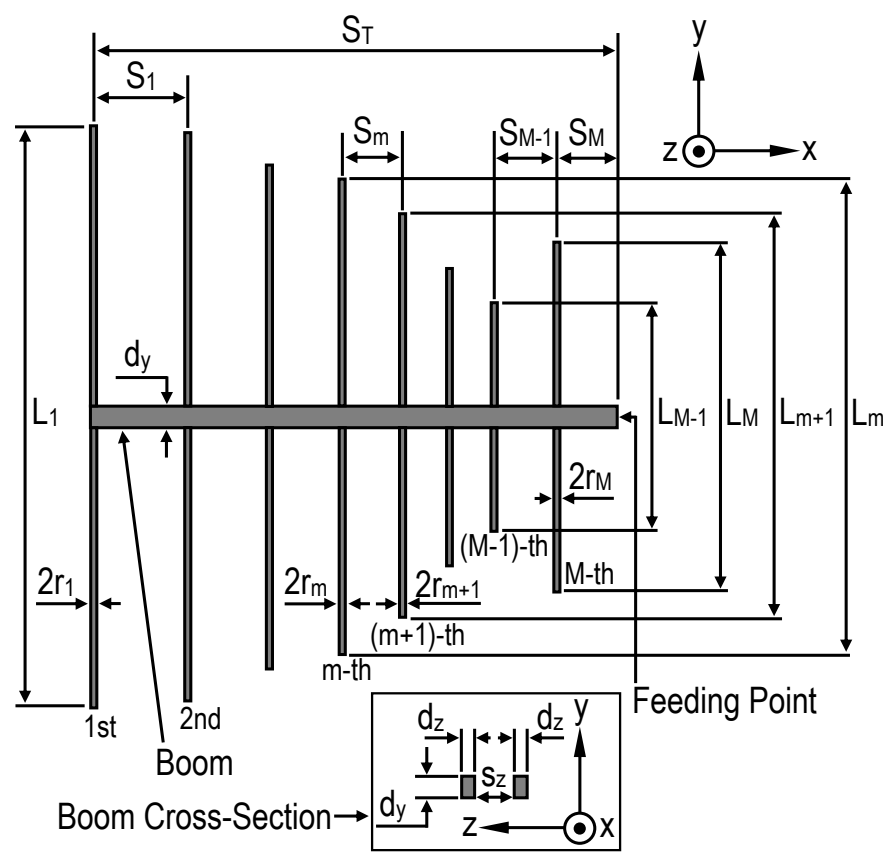

Fig. 1. Geometry of LTE-protected LPDA.

\section{PRIOR ART}

Evolutionary optimization methods have already been used as design methods of LPDAs especially in cases where the optimized antenna has to satisfy multiple requirements. Such cases are presented below. In some of these studies, the proposed method is compared to other methods in order to demonstrate its performance. Of course, Carrel's method can be used as a reference design method for any comparison. However, in all these studies, requirements have been set only for the operating band, without any requirement for signal rejection outside this band. To the best of the authors' knowledge, evolutionary optimization methods have never been applied to LPDAs so far, in order to improve signal reception inside a certain band and concurrently reject signals at frequencies outside this band.

An LPDA is optimized in [27] for operation in GSM, WiMAX, Bluetooth, Wi-Fi and 3G communications bands by using PSO in conjunction with the Method of Moments (MoM) [44]. The optimized antenna achieves values of $F G$ increased by $0.6-0.8 \mathrm{dBi}$ compared to respective values derived by Carrel's method, while $S W R$ is kept below 1.5.

A planar LPDA is optimized in [30] by applying PSO. The optimization is performed for operation in the S-band under requirements for maximum $F G$ and minimum $S W R$. The radiation characteristics are extracted here by using the FEKO software package. The optimized antenna achieves values of $F G$ between $8.5 \mathrm{dBi}$ and $10 \mathrm{dBi}$ and cross-polarization ratio below $-20 \mathrm{~dB}$ in the range $2-4 \mathrm{GHz}$.

In [34], PSO is applied in conjunction with the Numerical Electromagnetics Code (NEC) [45], [46], to perform a parametric study of LPDAs with respect to $F G$, the half power beam-widths (HPBWs) respectively on E-plane and H-plane, the front-to-back ratio $(F B R)$ and $S W R$. A 10-element LPDA is optimized for operation in the range $450-1350 \mathrm{MHz}$ under the following requirements: mean $F G \geq 8.2 \mathrm{dBi}$, mean

$F B R \geq 20 \mathrm{~dB}$ and mean $S W R \leq 1.5$.

In [36], PSOvm is applied in combination with CST MWS to optimize a 15-element LPDA for operation in the range 790-6000 MHz under the following requirements: $F G$ as high as possible, $G F \leq 2 \mathrm{~dB}$, secondary lobe level (SecLL) $\leq-20 \mathrm{~dB}$, and $S W R \leq 2$. These requirements are concurrently satisfied by proposing an exponential antenna geometry, where the dipole lengths and distances vary according to an exponential rule. It is noted that SecLL is a more general term than the side lobe level $(S L L)$ because it is defined as the highest level of all the secondary lobes (including side lobes and the back lobe) with respect to the main lobe peak gain.

In [39], IWO is applied in combination with NEC to optimize a 12-element LPDA for operation in the range 800$3300 \mathrm{MHz}$. The optimization is performed under the following requirements: $F G$ as high as possible, $G F \leq 2 \mathrm{~dB}$, $F B R \geq 20 \mathrm{~dB}, S L L \leq-20 \mathrm{~dB}$ on the E-plane, and $S W R \leq 1.8$. These requirements are concurrently satisfied by proposing an arbitrary LPDA geometry, where the dipole lengths and distances are independently optimized.

A Bacteria Foraging Algorithm (BFA) is applied in [47] to optimize LPDAs for operation in the analogue UHF TV band (470-870 MHz). The BFA aims at maximizing the average values of $F G$ and $F B R$ and minimizing the average values of $S W R$ and SLL over the operating band. Three antenna geometries, respectively composed of six, nine and twelve dipoles, are optimized. In these geometries, the dipoles are assumed to be inside the same angle as considered by Carrel's method and therefore the optimization variables are the parameters $\tau$ and $\sigma$ introduced by Carrel in [2].

Another LPDA optimization is performed in [48] for 
operation in WiMAX, GSM and Wi-Fi communication bands using a GA under requirements for high $F G$ and small antenna size. The optimized antenna achieves values of $F G$ improved by $0.6-1.7 \mathrm{dBi}$ compared to respective values derived by Carrel's method, while the antenna size is reduced by $12 \%$.

In [49], a GA is used to optimize a miniaturized inverted-V LPDA, composed of thin metal wires mounted over lossy ground. The NEC2 software is employed by the GA to calculate the antenna radiation characteristics. The LPDA is optimized in the range 6-30 $\mathrm{MHz}$ under the following requirements: $\quad S L L \leq-6 \mathrm{~dB}, \quad S W R \leq 1.5, \quad F G \geq 8 \mathrm{dBi}$ and small size.

In [50], a circular switched parasitic array of LPDAs is optimized for operation in the range $3.1-10.6 \mathrm{GHz}$ by using a GA. The optimization procedure aims at maximizing $F G$ and minimizing $S W R$ with desired values equal to $8 \mathrm{dBi}$ and 1 , respectively. The circular array of LPDAs provides the ability for beam-steering by setting the appropriate LPDA to be connected to the signal source, while the other LPDAs operate as parasitic antennas. An optimal geometry of a circular array of four 12-element LPDAs is presented. The radiation characteristics of the whole structure are extracted by applying the SuperNEC software.

A multi-objective optimization of LPDAs is performed in [51] by applying the Non-dominated Sorting Genetic Algorithm II (NSGA-II). The algorithm aims at maximizing $F G$ over the range $3-30 \mathrm{MHz}$ while minimizing $S W R$ over the same range and the total LPDA length. The antenna radiation characteristics are extracted by employing the Graphic Numerical Electromagnetics Code (GNEC).

In [52] and [53], a GA is applied in conjunction with MoM as LPDA design method. The optimization procedure aims at minimizing the total LPDA length as well as the number of dipoles that compose the LPDA while preserving the average values of $F G$ and $S W R$ over the operating band.

Finally, a GA, the Nelder-Mead Downhill Simplex method, and a combined GA/Nelder-Mead method are applied in [54] to maximize the average $F G$, and also minimize $G F$, the average $S W R$ and the standard deviation of $S W R$ over the operating band. A 7-element LPDA and a 20-element LPDA are optimized by the above three methods respectively in the ranges $800-1600 \mathrm{MHz}$ and $200-1300 \mathrm{MHz}$. To extract the radiation characteristics of both LPDAs, the optimizers employ NEC. The comparison shows that the combined GA/Nelder-Mead method outperforms both GA and NelderMead method. However, all three methods achieve better LPDAs than the LPDA extracted by Carrel's method.

\section{PSOVM}

Evolutionary optimization has widely been used to find optimal solutions in many problems of telecommunications and electromagnetics. PSO and its variants belong to a great category of evolutionary programming, which is based on swarm intelligence. Two major versions of PSO have been proposed so far: the Inertia Weight based PSO (IWPSO) and the Constriction Coefficient based PSO (CCPSO) [32]. The difference between IWPSO and CCPSO concerns the update formula of the particle velocity. Both variants may utilize either the Global Best (gbest) experience model, where a particle is permitted to exchange information with any particle of the swarm, or the Local Best (lbest) experience model, where a particle is permitted to exchange information only inside its topological neighborhood [32].

PSOvm is a variant of PSO based on the gbest model of CCPSO. According to PSOvm, $N$ particles are disseminated in a search space of $D$ dimensions ( $D$ is the number of independent variables to be optimized), and therefore their velocities and positions are respectively updated during the $i$ th iteration $(i=1, \ldots, I)$ of the optimization process as follows:

$$
\begin{array}{r}
v_{n d}(i+1)=k\left\{v_{n d}(i)+\varphi_{1} r\left[p_{n d}(i)-x_{n d}(i)\right]\right. \\
\left.+\varphi_{2} r\left[g_{d}(i)-x_{n d}(i)\right]\right\} \\
x_{n d}(i+1)=x_{n d}(i)+v_{n d}(i+1)
\end{array}
$$

In the above expressions:

- $v_{n d}(i)$ and $v_{n d}(i+1)$ are respectively the $d$-th velocity components $(d=1, \ldots, D)$ of the $n$-th particle $(n=1, \ldots, N)$ of the swarm during the current $(i$-th) and the next $((i+1)$ th) iteration,

- $x_{n d}(i)$ and $x_{n d}(i+1)$ are respectively the $d$-th position coordinates of the $n$-th particle during the current and the next iteration,

- $\quad p_{n d}(i)$ and $g_{d}(i)$ are the $d$-th coordinates of the best positions found respectively by the $n$-th particle and the whole swarm at the end of the current iteration,

- $\varphi_{1}$ and $\varphi_{2}$ are two coefficients introduced in CCPSO, which reflect respectively the self-exploration of every particle and the exploitation of the swarm knowledge, and are both equal to 2.05 as explained in [32],

- $k$ is the constriction coefficient also introduced in CCPSO and considered to be equal to 0.73 as explained in [32], and finally

- $r$ are random numbers uniformly distributed in the interval $(0,1)$ - a random number generator is employed to produce such a number for every iteration, particle and dimension.

In the LPDA optimization problem studied here, the position coordinates are the 22 optimization variables $(D=22)$

described in the last paragraph of Section II. Therefore, the position vector has the following form:

$\left[x_{n 1} \ldots x_{n 22}\right]=\left[L_{1} \ldots L_{10} S_{1} \ldots S_{10} d_{y} s_{z}\right]$

It is expected that the velocity update as given by (1) will improve the particles' positions (or equivalently the particles' fitness values). However, a particle's position may not be improved at the end of an iteration. In such a case, the velocity update (which is going to be performed in the next iteration) will probably not improve the particle's position, if we use the 
velocity components of this particle exactly as calculated during the current iteration. In other words, it is not efficient to use (1) in such a case. On the contrary, the probability to improve the particle's position greatly increases, if we apply a perturbation to the velocity components. This idea is implemented by a particular mechanism called velocity mutation and it is the main technique proposed in PSOvm. So, let's assume that, at the end of $i$-th iteration, the $n$-th particle does not manage to achieve a better fitness. Then, its velocity components are mutated by multiplying them by the factor

$F_{j}=(0.1 j+0.6)(2 r-1), \quad j=1, \ldots, 6$,

where $j$ is the number of iterations in a row with no position improvement (or fitness improvement) for this particle. In such a case, the velocity update is given by:

$$
\begin{aligned}
v_{n d}(i+1) & =k\left\{F_{j} v_{n d}(i)+\varphi_{1} r\left[p_{n d}(i)-x_{n d}(i)\right]\right. \\
& \left.+\varphi_{2} r\left[g_{d}(i)-x_{n d}(i)\right]\right\}, \quad j=1, \ldots, 6
\end{aligned}
$$

The position for such a particle is updated again by (2).

The form of $F_{j}$ has empirically been extracted after many trials, where PSOvm was applied on a large number of benchmark functions. The results from these trials are summarized below:

1. If only one failure of position improvement occurs $(j=1)$, then the highest probability to improve the particle's position in the next iteration is recorded when $F_{j}$ is uniformly distributed in the interval $(-0.7,+0.7)$.

2. For every additional failure in a row, it is better to symmetrically broaden the above interval by 0.2 , i.e., the interval becomes $(-0.8,+0.8)$ for the next failure, $(-0.9$, +0.9 ) for the next one, etc.

3. The mutation process is better to be performed for up to six failures in a row $(j=1, \ldots, 6$ in (4) or (5)) for the sake of convergence speed. Consequently, the mutation process is no longer repeated after six failures in a row or after a position improvement. Then, in the next iteration, the particle's velocity is updated by applying (1).

PSOvm utilizes the same restrictions on particle velocities and positions as used in CCPSO [32]. So, $v_{n d}(i)$ is limited by a maximum value $\left(\operatorname{vmax}_{d}\right)$, which is defined to be equal to $15 \%$ of the width of the search space along the $d$-th dimension, i.e., if $v_{n d}(i)>v_{\max }$ then $v_{n d}(i)=v \max _{d}$, and also if $v_{n d}(i)<-\operatorname{vmax}_{d}$ then $v_{n d}(i)=-v_{\max }$, where

$$
\operatorname{vmax}_{d}=0.15\left(\max _{d}-x \min _{d}\right)
$$

It is noted that xmax $_{d}$ and $\mathrm{xmin}_{d}$ are the boundaries of the position coordinates along the $d$-th dimension of the search space. In addition, PSOvm adopts the absorbing walls condition to confine the particles within the search space.

\section{FITNESS FunCTION DEFINITION}

Since multiple requirements have been set on the antenna design, the antenna optimization is an inherently multi-target problem. On the other hand, PSOvm and many other evolutionary optimization methods aim at finding the nearglobal optimum (i.e., minimum in our optimization problem) of a single mathematical function fit, which is called fitness function (already mentioned above). Consequently, fit must be defined here as a linear combination of four terms respectively formulated according to the four design requirements described in Section II. When fit reaches its global minimum, all the terms that compose fit come up to their respective minimum values and thus all the requirements are finally satisfied. Therefore, fit can be defined as follows:

$$
\begin{aligned}
f i t & =\max \left(S W R_{\max }^{P B}, 1.9\right)+\left(-F G_{\min }^{P B}\right) \\
& +\max \left(G F^{P B}, 2.5\right)+\max \left(F G_{\max }^{S B}, 0\right)
\end{aligned}
$$

In the above formula, $S W R_{\text {max }}^{P B}, F G_{\text {min }}^{P B}$ and $G F^{P B}$ are respectively the maximum $S W R$, the minimum $F G$ in $\mathrm{dBi}$ and $G F$ in $\mathrm{dB}$, all found inside the passband (470-780 MHz), while $F G_{\max }^{S B}$ is the maximum $F G$ in $\mathrm{dBi}$ found inside the stopband (800-900 MHz). To find the above values, $S W R$ and $F G$ are calculated at steps of $10 \mathrm{MHz}$ inside the ranges $470-780 \mathrm{MHz}$ and $800-900 \mathrm{MHz}$. Therefore, the antenna is analyzed at steps of $10 \mathrm{MHz}$ inside the above two ranges by applying CST MWS [43]. The calculation of $S W R$ is performed by assuming a $50 \mathrm{Ohm}$ transmission line that feeds the LPDA.

Minimization of the 2nd term $\left(-F G_{\min }^{P B}\right)$ results in maximization of $F G$ (due to the "minus" sign of the term) over the entire passband. The other three terms are formulated in such a way that values of $S W R_{\max }^{P B}, G F^{P B}$ and $F G_{\text {max }}^{S B}$ respectively less than $1.9,2.5 \mathrm{~dB}$ and $0 \mathrm{dBi}$ do not cause further minimization of fit, since the respective requirements have already been satisfied.

\section{DEFINITION OF LiMITS OF OPTIMIZATION VARIABLES}

Due to the large number of optimization variables, the search space of every variable must be restricted between a lower and an upper limit, to help all the optimization methods find optimal results. The limits of the dipole lengths and distances can be estimated by considering that the optimized values of lengths and distances will deviate at most $30 \%$ from the respective typical values derived by Carrel's method. In particular, from the constant directivity contour curves (corrected Carrel's graph, [1]) and considering antenna directivity equal to $7.5 \mathrm{dBi}$, the optimum value of $\sigma$ is derived equal to 0.158 and the respective value of $\tau$ equal to 0.862 . From these two values and since the passband extends from $470 \mathrm{MHz}$ to $780 \mathrm{MHz}$, Carrel's method extracts an LPDA composed of 9 dipoles. The largest dipole $(m=1)$ should be in resonant condition at the lowest frequency 
( $\left.f_{\min }=470 \mathrm{MHz}\right)$ of the passband, and therefore its length must be

$L_{1, \text { Carrel }}=\lambda_{\max } / 2$

where $\lambda_{\max }$ is the wavelength at $470 \mathrm{MHz}$. Then, the lengths of the rest of the dipoles and the distances between adjacent dipoles are calculated according to Carrel's method by using the following respective expressions:

$$
\begin{array}{ll}
L_{m+1, \text { Carrel }}=\tau L_{m, \text { Carrel }}, & m=1, \ldots, 8 \\
S_{m, \text { Carrel }}=2 \sigma L_{m, \text { Carrel }}, & m=1, \ldots, 8
\end{array}
$$

By assuming a deviation of $30 \%$ from the above Carrel-based values as previously mentioned, the limits of the dipole lengths and distances are estimated as follows:

$$
\begin{aligned}
& L_{m, \min }=0.7 L_{m, \text { Carrel }}, \quad m=1, \ldots, 9 \\
& L_{m, \max }=1.3 L_{m, \text { Carrel }}, \quad m=1, \ldots, 9 \\
& S_{m, \text { min }}=0.7 S_{m, \text { Carrel }}, \quad m=1, \ldots, 8 \\
& S_{m, \max }=1.3 S_{m, \text { Carrel }}, \quad m=1, \ldots, 8
\end{aligned}
$$

Nevertheless, one more dipole $(m=10)$ should be used in front of the previous 9 dipoles, explaining at this point why we decided to use 10 dipoles $(M=10)$ to build the LTEprotected LPDA. This extra dipole is going to be used as a reflector for frequencies greater than $780 \mathrm{MHz}$ and thus make $F G$ decrease outside the passband, as described in Section II. To do so, this dipole must be greater in length than the previous (ninth) one. Therefore, it is reasonable to assume that the lower length limit of the tenth dipole coincides with the upper length limit of the ninth one, as given by the following expression:

$$
L_{10, \min }=L_{9, \max }=1.3 L_{9, \text { Carrel }}
$$

Also, the upper length limit of the tenth dipole is arbitrarily considered to be $30 \%$ greater than $L_{10, \min }$, as given by:

$$
L_{10, \max }=1.3 L_{10, \min }
$$

Since ten dipoles are used in the LTE-protected LPDA geometry, two more distances, $S_{9}$ and $S_{10}$, have to be defined (see Fig. 1). The first one is the distance between the ninth and the tenth dipole, while the second is the distance between the tenth dipole and the feeding point of the antenna. To avoid contact between the ninth and the tenth dipole, a condition has to be set for $S_{9}$ as follows:

$$
S_{9}>r_{9}+r_{10}=4 \mathrm{~mm}
$$

Due to the thickness of the dipoles, the feeding must be applied at a distance greater than or equal to the radius of the tenth dipole, as given by the following condition:

$S_{10} \geq r_{10}=2 \mathrm{~mm}$

Due to (17) and (18), the lower limits of $S_{9}$ and $S_{10}$ are respectively set as follows:

$S_{9, \text { min }}=r_{9}+r_{10}+1 \mathrm{~mm}=5 \mathrm{~mm}$

$S_{10, \text { min }}=r_{10}=2 \mathrm{~mm}$

The upper limits of $S_{9}$ and $S_{10}$ are both set equal to $\lambda_{\min } / 4$, where $\lambda_{\min }$ is the wavelength at the highest frequency ( $\left.f_{\max }=780 \mathrm{MHz}\right)$ of the passband, considering that the maximum variation of voltage, current or impedance is observed along a quarter of the wavelength. Therefore:

$S_{9, \max }=S_{10, \max }=\lambda_{\min } / 4=0.0962 \mathrm{~m}$

The dipole length limits are shown in Table I, while the dipole distance limits are shown in Table II.

Finally, the values of both $d_{y}$ and $s_{z}$ are restricted between $1 \mathrm{~mm}$ and $1 \mathrm{~cm}$. Therefore:

$d_{y, \min }=s_{z, \min }=1 \mathrm{~mm}$
$d_{y, \max }=s_{z, \max }=1 \mathrm{~cm}$

TABLE I

\begin{tabular}{cccc}
\multicolumn{4}{c}{ CARREL-BASED DIPOLE LENGTHS AND DIPOLE LENGTH LIMITS } \\
\hline$m$ & $L_{m, \text { Carrel }}$ (meters) & $L_{m, \min }($ meters $)$ & $L_{m, \max }($ meters $)$ \\
\hline 1 & 0.3191 & 0.2234 & 0.4149 \\
2 & 0.2751 & 0.1926 & 0.3576 \\
3 & 0.2371 & 0.1660 & 0.3083 \\
4 & 0.2044 & 0.1431 & 0.2657 \\
5 & 0.1762 & 0.1233 & 0.2291 \\
6 & 0.1519 & 0.1063 & 0.1975 \\
7 & 0.1309 & 0.0917 & 0.1702 \\
8 & 0.1129 & 0.0790 & 0.1467 \\
9 & 0.0973 & 0.0681 & 0.1265 \\
10 & - & 0.1265 & 0.1644 \\
\hline
\end{tabular}

TABLE II

CARREl-BASEd Dipole DistanCES AND Dipole Distance Limits

\begin{tabular}{cccc}
\hline$m$ & $S_{m, \text { Carrel }}$ (meters) & $S_{m, \min }($ meters $)$ & $S_{m, \max }$ (meters) \\
\hline 1 & 0.1009 & 0.0706 & 0.1311 \\
2 & 0.0869 & 0.0609 & 0.1130 \\
3 & 0.0749 & 0.0525 & 0.0974 \\
4 & 0.0646 & 0.0452 & 0.0840 \\
5 & 0.0557 & 0.0390 & 0.0724 \\
6 & 0.0480 & 0.0336 & 0.0624 \\
7 & 0.0414 & 0.0290 & 0.0538 \\
8 & 0.0357 & 0.0250 & 0.0464 \\
9 & - & 0.0050 & 0.0962 \\
10 & - & 0.0020 & 0.0962 \\
\hline
\end{tabular}

\section{COMPARATIVE RESUlts}

All methods compared in this paper employ populations of 20 particles $(N=20)$ in order to estimate the values of the 22 optimization variables $(D=22)$, which have been described 
in the last paragraph of Section II. By applying PSOvm on the LPDA optimization problem several times, it was found that a final stable fitness value is achieved after 2000 evaluations of fitness function. A fair comparison among all optimization methods (PSOvm, CCPSO, DE, IWO and GA) demands the same total number of fitness function evaluations per execution for every method. Also, due to its stochastic nature, any of these methods achieves a different final fitness value, each time the method is executed. To reveal its real potential, the method must be executed several times - in fact 10 times. Each execution is completed after 2000 fitness function evaluations. Then, we choose the execution that corresponds to the best (i.e., lowest) final fitness value, because this execution reflects the best performance of the method. To illustrate the best performance of the method, this execution is recorded. Therefore, for every iteration of the execution, we record the number of fitness evaluations performed and the lowest fitness value achieved up to this iteration. The variation of the fitness values with respect to the number of fitness evaluations is illustrated as best performance graph and is derived for every optimization method. These graphs are given in Fig. 2. It is obvious that the final fitness value achieved by PSOvm is better (i.e., lower) than the respective values achieved by the rest of the methods. This means that the optimized LPDA geometry found by PSOvm is closer to the predefined requirements than the respective geometries found by CCPSO, DE, IWO and GA. It is also noteworthy that PSOvm converges faster than the other methods in the first iterations. The values of the geometry parameters that define the PSOvm-based LPDA are given in Table III.

By adding all the distances $S_{m}, m=1, \ldots, 10$, the antenna length $S_{T}$ (see Fig. 1) is derived equal to $0.619 \mathrm{~m}$. Then, we apply Carrel's method, [1], in order to design an LPDA that has the same length as the optimized antenna given above and operates in the same frequency range $(470-780 \mathrm{MHz})$. In particular, from the constant directivity contour curves (corrected Carrel's graph, [1]) and considering antenna length equal to $0.619 \mathrm{~m}$, the optimum value of $\sigma$ is derived equal to 0.1648 and the respective value of $\tau$ equal to 0.8891 , while the derived antenna has to be composed of 10 dipoles. The largest dipole $(m=1)$ is considered to be in resonant condition at the lowest frequency $\left(f_{\min }=470 \mathrm{MHz}\right)$ of the passband, and therefore its length must be equal to $\lambda_{\max } / 2$, as shown in (8). Also, we decided to set the radius of the shortest dipole $(m=10)$ equal to the radius $(2 \mathrm{~mm})$ of the dipoles of the optimized LPDA, and therefore the other (larger) dipoles of the LPDA will have larger radii, resulting thus in an antenna that can be fabricated in practice. So, the rest of the lengths and radii as well as the dipole distances are calculated according to the well-known expressions given below:

$$
\begin{array}{ll}
L_{m+1}=\tau L_{m}, & m=1, \ldots, 9 \\
r_{m}=r_{m+1} / \tau, & m=9, \ldots, 1 \\
S_{m}=2 \sigma L_{m}, & m=1, \ldots, 9
\end{array}
$$

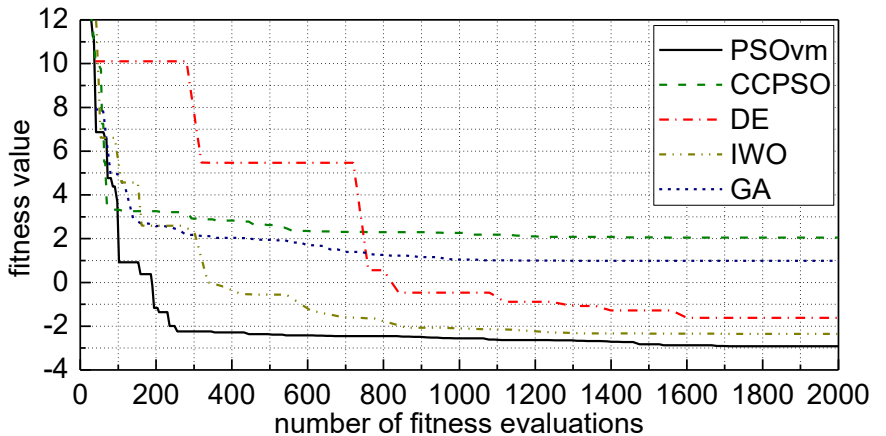

Fig. 2. Best performance graphs of PSOvm, CCPSO, DE, IWO and GA.

TABLE III

PSOVM-BASED LTE-PROTECTED LPDA

\begin{tabular}{ccc}
\hline$m$ & $L_{m}$ (meters) & $S_{m}$ (meters) \\
\hline 1 & 0.363 & 0.087 \\
2 & 0.288 & 0.082 \\
3 & 0.240 & 0.097 \\
4 & 0.222 & 0.069 \\
5 & 0.167 & 0.053 \\
6 & 0.151 & 0.052 \\
7 & 0.144 & 0.044 \\
8 & 0.099 & 0.031 \\
9 & 0.088 & 0.079 \\
10 & 0.149 & 0.025 \\
\hline$d_{y}=5 \mathrm{~mm}$ & $s_{z}=4 \mathrm{~mm}$ \\
$d_{z}=4 \mathrm{~mm}$ (fixed) & $r_{m}=2 \mathrm{~mm}$ (fixed) $, m=1, \ldots, 10$ \\
\hline
\end{tabular}

Finally, the values of $L_{1} / r_{1}$ and $\sigma / \sqrt{\tau}$ are used to estimate the characteristic impedance $Z_{0}$ of the transmission line that simulates the boom of the LPDA. In this way, we get $Z_{0}=60$ $\mathrm{Ohm}$. In order to have a realistic antenna geometry, we have to find the physical dimensions of a boom that corresponds to the above characteristic impedance. According to [55]-[57] and considering that the boom consists of two parallel square rods with $s_{z}=4 \mathrm{~mm}$ (i.e., the same spacing between the closest surfaces of the rods as that of the optimized LPDA), the side length of the square cross section of the rods is derived to be equal to $15.7 \mathrm{~mm}$ (i.e., $d_{y}=d_{z}=15.7 \mathrm{~mm}$ ). All these values of Carrel's geometry are shown in Table IV.

TABLE IV CARREL'S LPDA GEOMETRY

\begin{tabular}{cccc}
\hline$m$ & $L_{m}$ (meters) & $S_{m}$ (meters) & $r_{m}$ (meters) \\
\hline 1 & 0.3191 & 0.1052 & 0.0058 \\
2 & 0.2838 & 0.0935 & 0.0051 \\
3 & 0.2523 & 0.0831 & 0.0046 \\
4 & 0.2243 & 0.0739 & 0.0040 \\
5 & 0.1994 & 0.0657 & 0.0036 \\
6 & 0.1773 & 0.0584 & 0.0032 \\
7 & 0.1577 & 0.0520 & 0.0028 \\
8 & 0.1402 & 0.0462 & 0.0025 \\
9 & 0.1246 & 0.0411 & 0.0022 \\
10 & 0.1108 & - & 0.0020 \\
\hline & $Z_{0}=60 \mathrm{Ohm}$ & $S_{z}=4 \mathrm{~mm}$ & $d_{y}=d_{z}=15.7 \mathrm{~mm}$ \\
\hline
\end{tabular}

Then, CST MWS is applied on Carrel's LPDA geometry (shown in Table IV) to calculate $S W R, F G$ and realized gain $(R G)$ [58] over the range $450-900 \mathrm{MHz}$ and thus examine the behavior of this geometry inside and outside the passband. This behavior is illustrated in comparison to the respective 
behavior of the PSOvm-based LPDA in the graphs of Figs. 35. It is noted that $R G$ is equal to $F G$ reduced by the losses due to the mismatch of the antenna input impedance to the characteristic impedance $(50 \mathrm{Ohm})$ of the transmission line that feeds the antenna, as shown in the expression

$$
R G[\mathrm{dBi}]=F G[\mathrm{dBi}]+10 \log \left(1-|\rho|^{2}\right),
$$

where $|\rho|$ is the absolute value of the complex reflection coefficient at the antenna input. The value of $|\rho|$ is estimated from $S W R$ for each frequency as follows:

$$
|\rho|=\frac{S W R-1}{S W R+1}
$$

The graphs of Figs. 3-5 are utilized to estimate the minimum, the maximum and the average value of $S W R, F G$ and $R G$, as well as $G F$ inside the passband $(470-780 \mathrm{MHz})$, and finally the minimum, the maximum and the average value of $F G$ and $R G$ inside the stopband (800-900 MHz). The results are shown in Table V.

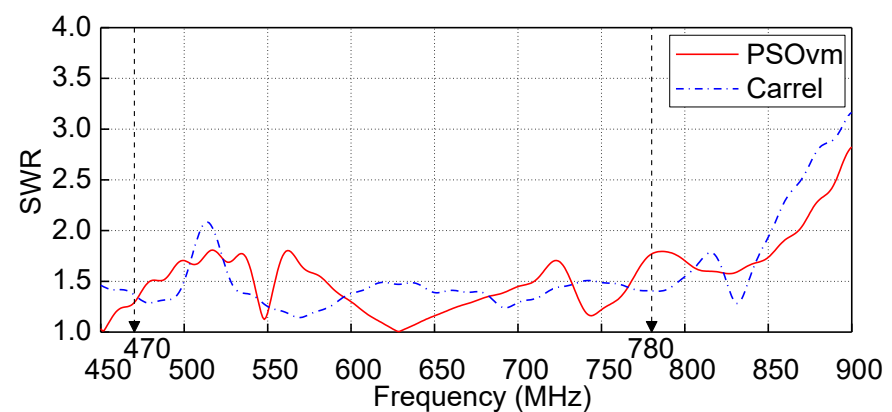

Fig. 3. Comparative graphs of SWR vs. frequency of the PSOvm-based LPDA and Carrel's LPDA.

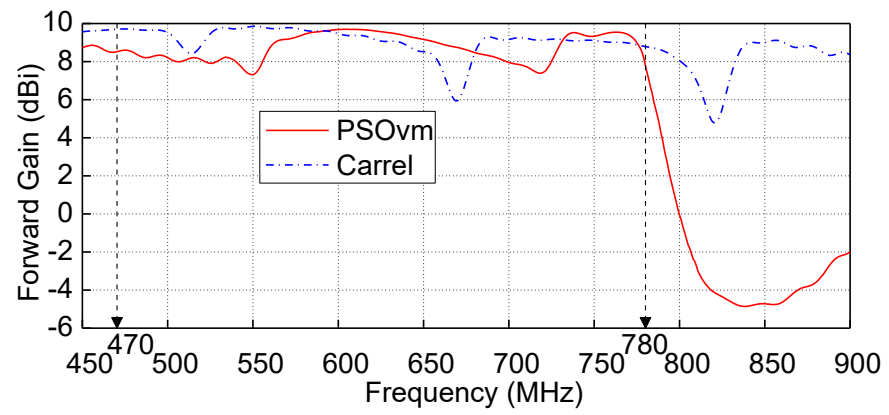

Fig. 4. Comparative graphs of FG vs. frequency of the PSOvm-based LPDA and Carrel's LPDA.

It seems that PSOvm and Carrel's method exhibit almost similar performance in terms of $S W R$, and also both methods satisfy the impedance matching condition. Moreover, the average values of $F G$ and $R G$ inside the passband achieved by Carrel's LPDA are only $0.38 \mathrm{~dB}$ and $0.39 \mathrm{~dB}$ higher than the respective values obtained by the PSOvm-based LPDA. However, Carrel's LPDA seems to exhibit greater fluctuations in $F G$ (and $R G$ ) resulting thus in $1.55 \mathrm{~dB}$ higher $G F$ compared to the PSOvm-based LPDA, which achieves $G F \leq 2.5 \mathrm{~dB}$ according to the respective (3rd) requirement. After all, the most important conclusion is that the 4th requirement $(F G \leq$ $0 \mathrm{dBi}$ inside the stopband) is satisfied only by the PSOvmbased LPDA, which means that the ability to reject LTE800 signals is well provided by the PSOvm-based LPDA and not at all by Carrel's LPDA.

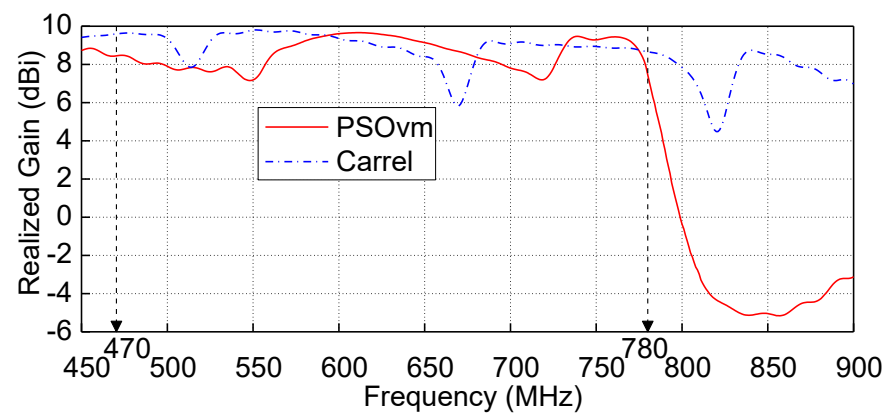

Fig. 5. Comparative graphs of RG vs. frequency of the PSOvm-based LPDA and Carrel's LPDA.

TABLE V

ANTENNA PERFORMANCE PARAMETERS

\begin{tabular}{ccc}
\multicolumn{2}{c}{ ANTENNA PERFORMANCE PARAMETERS } \\
\hline Performance & PSOvm & Carrel \\
Parameter & & \\
\hline PASSBAND: & 1.01 & 1.14 \\
Minimum $S W R$ & 1.81 & 2.08 \\
Maximum $S W R$ & 1.43 & 1.41 \\
Average $S W R$ & 7.32 & 5.93 \\
Minimum $F G(\mathrm{dBi})$ & 9.69 & 9.85 \\
Maximum $F G(\mathrm{dBi})$ & 8.74 & 9.12 \\
Average $F G(\mathrm{dBi})$ & 2.37 & 3.92 \\
$G F(\mathrm{~dB})$ & 7.16 & 5.81 \\
Minimum $R G(\mathrm{dBi})$ & 9.66 & 9.81 \\
Maximum $R G(\mathrm{dBi})$ & 8.59 & 8.98 \\
Average $R G(\mathrm{dBi})$ & & \\
\hline STOPBAND: & -4.86 & 4.77 \\
Minimum $F G(\mathrm{dBi})$ & -0.07 & 9.12 \\
Maximum $F G(\mathrm{dBi})$ & -3.68 & 8.01 \\
Average $F G(\mathrm{dBi})$ & -5.16 & 4.48 \\
Minimum $R G(\mathrm{dBi})$ & -0.37 & 8.74 \\
Maximum $R G(\mathrm{dBi})$ & -4.14 & 7.40 \\
Average $R G(\mathrm{dBi})$ & &
\end{tabular}

\section{CONCLUSIONS}

In comparison to CCPSO, DE, IWO and GA, PSOvm seems to be able to obtain better fitness values and thus come closer to multiple requirements defined over the passband and the stopband. Especially, due to its ability to achieve low $F G$ in the stopband, i.e., for frequencies above $800 \mathrm{MHz}$, PSOvm becomes a remarkable optimization tool, useful to design LPDAs that reject LTE800 signals. Thus, the signal reception is improved in the DVB-T band without the need of using an external LTE-band rejection filter. Also, due to its higher convergence rate in the first iterations, PSOvm approaches the final result in less fitness function evaluations than the above four methods. The PSOvm-based LPDA achieves similar $S W R, F G$ and $R G$ values but much better (lower) $G F$ in the passband compared to Carrel's LPDA. Finally, the requirement for LTE rejection is fully satisfied by PSOvm, while Carrel's method totally fails. 


\section{REFERENCES}

[1] C. A. Balanis, "Frequency independent antennas, antenna miniaturization, and fractal Antennas," in Antenna Theory, Analysis and Design, 3rd ed., New Jersey: John Wiley \& Sons, 2005, pp. 611-652.

[2] R. L. Carrel, "Analysis and design of the log-periodic dipole antenna," $\mathrm{Ph} . D$. dissertation, Dept. Elect. Eng., University of Illinois, University Microfilms, Inc., Ann Arbor, MI, 1961.

[3] P. C. Butson and G. T. Thompson, "A note on the calculation of the gain of log-periodic dipole antennas," IEEE Trans. Antennas Propagat., vol. AP-24, no. 1, pp. 105-106, Jan. 1976.

[4] R. Cox. (2016, July). The W8IO Antenna Site - LPCAD software. [Online]. Available: http://www.w8io.com/LPCAD.htm

[5] R. Cox. (2016, March). The W8IO Antenna Site - LPCAD Example Antennas. [Online]. Available: http://w8io.com/LPCAD-Examples.htm

[6] T. Pavlenko, C. Reustle, Y. Dobrev, M. Gottinger, L. Jassoume, and M. Vossiek, "Design and optimization of sparse planar antenna arrays for wireless 3D local positioning systems," IEEE Trans. Antennas Propagat., to be published.

[7] M. A. Rodriguez Barrera and W. P. Carpes, "Bandwidth for the equivalent circuit model in square-loop frequency selective surfaces," IEEE Trans. Antennas Propagat., vol. 65, no. 11, pp. 5932-5939, Nov. 2017.

[8] M. Kovaleva, D. Bulger, B. A. Zeb, and K. P. Esselle, "Cross-entropy method for electromagnetic optimization with constraints and mixed variables," IEEE Trans. Antennas Propagat., vol. 65, no. 10, pp. 55325540, Oct. 2017.

[9] X. Ding, Y. F. Cheng, W. Shao, and B. Z. Wang, "A wide-angle scanning phased array with microstrip patch mode reconfiguration technique," IEEE Trans. Antennas Propagat., vol. 65, no. 9, pp. 45484555, Sept. 2017.

[10] B. Babakhani and S. K. Sharma, "Dual null steering and limited beam peak steering using triple-mode circular microstrip patch antenna," IEEE Trans. Antennas Propagat., vol. 65, no. 8, pp. 3838-3848, Aug. 2017.

[11] S. Jayaprakasam, S. K. Abdul Rahim, C. Y. Leow, T. O. Ting, and A. A. Eteng, "Multiobjective beampattern optimization in collaborative beamforming via NSGA-II with selective distance," IEEE Trans. Antennas Propagat., vol. 65, no. 5, pp. 2348-2357, May 2017.

[12] Y. F. Cheng, X. Ding, W. Shao, and B. Z. Wang, "Planar wide-angle scanning phased array with pattern-reconfigurable windmill-shaped loop elements," IEEE Trans. Antennas Propagat., vol. 65, no. 2, pp. 932936, Feb. 2017.

[13] D. Caratelli, L. Mescia, P. Bia, and O. V. Stukach, "Fractional-calculusbased FDTD algorithm for ultrawideband electromagnetic characterization of arbitrary dispersive dielectric materials," IEEE Trans. Antennas Propagat., vol. 64, no. 8, pp. 3533-3544, Aug. 2016.

[14] K. Eldridge, A. Fierro, J. Dickens, and A. Neuber, "A take on arbitrary transient electric field reconstruction using wavelet decomposition theory coupled with particle swarm optimization," IEEE Trans. Antennas Propagat., vol. 64, no. 7, pp. 3151-3159, July 2016.

[15] C. H. Jang, F. Hu, F. He, J. Li, and D. Zhu, "Low-redundancy large linear arrays synthesis for aperture synthesis radiometers using particle swarm optimization," IEEE Trans. Antennas Propagat., vol. 64, no. 6, pp. 2179-2188, June 2016.

[16] G. G. Lema, G. T. Tesfamariam, and M. I. Mohammed, "A novel elliptical-cylindrical antenna array for radar applications," IEEE Trans. Antennas Propagat., vol. 64, no. 5, pp. 1681-1688, May 2016.

[17] A. H. Abdelrahman, P. Nayeri, A. Z. Elsherbeni, and F. Yang, "Singlefeed quad-beam transmitarray antenna design," IEEE Trans. Antennas Propagat., vol. 64, no. 3, pp. 953-959, March 2016.

[18] D. Sun, Z. Zhang, X. Yan, and X. Jiang, "Design of broadband dualpolarized patch antenna with backed square annular cavity," IEEE Trans. Antennas Propagat., vol. 64, no. 1, pp. 43-52, Jan. 2016.

[19] L. Guo and A. M. Abbosh, "Optimization-based confocal microwave imaging in medical applications," IEEE Trans. Antennas Propagat., vol. 63, no. 8, pp. 3531-3539, Aug. 2015.

[20] J. M. Kovitz, H. Rajagopalan, and Y. Rahmat-Samii, "Design and implementation of broadband MEMS RHCP/LHCP reconfigurable arrays using rotated E-shaped patch elements," IEEE Trans. Antennas Propagat., vol. 63, no. 6, pp. 2497-2507, June 2015.

[21] G. Oliveri, F. Viani, N. Anselmi, and A. Massa, "Synthesis of multilayer WAIM coatings for planar-phased arrays within the system-by-design framework," IEEE Trans. Antennas Propagat., vol. 63, no. 6, pp. 24822496, June 2015.
[22] W. Xu, B. Y. Duan, P. Li, N. Hu, and Y. Qiu, "Multiobjective particle swarm optimization of boresight error and transmission loss for airborne radomes," IEEE Trans. Antennas Propagat., vol. 62, no. 11, pp. 58805885, Nov. 2014.

[23] A. Deb, J. S. Roy, and B. Gupta, "Performance comparison of differential evolution, particle swarm optimization and genetic algorithm in the design of circularly polarized microstrip antennas," IEEE Trans. Antennas Propagat., vol. 62, no. 8, pp. 3920-3928, Aug. 2014.

[24] S. Kibria, M. T. Islam, and B. Yatim, "New compact dual-band circularly polarized universal RFID reader antenna using ramped convergence particle swarm optimization," IEEE Trans. Antennas Propagat., vol. 62, no. 5, pp. 2795-2801, May 2014.

[25] T. H. Nguyen, H. Morishita, Y. Koyanagi, K. Izui, and S. Nishiwaki, "A multi-level optimization method using PSO for the optimal design of an L-shaped folded monopole antenna array," IEEE Trans. Antennas Propagat., vol. 62, no. 1, pp. 206-215, Jan. 2014.

[26] S. H. Yang and J. F. Kiang, "Adjustment of beamwidth and side-lobe level of large phased-arrays using particle swarm optimization technique," IEEE Trans. Antennas Propagat., vol. 62, no. 1, pp. 138144, Jan. 2014

[27] M. Aziz-ul-Haq, M. Tausif Afzal, Umair Rafique, Qamar-ud-Din, M. Arif Khan, and M. Mansoor Ahmed, "Log periodic dipole antenna design using particle swarm optimization," Int. J. Electromagnetics and Applications, vol. 2, no. 4, pp. 65-68, 2012.

[28] R. Bhattacharya, T. K. Bhattacharyya, and R. Garg, "Position mutated hierarchical particle swarm optimization and its application in synthesis of unequally spaced antenna arrays," IEEE Trans. Antennas Propag., vol. 60, no. 7, pp. 3174-3181, July 2012.

[29] A. Modiri and K. Kiasaleh, "Modification of real-number and binary PSO algorithms for accelerated convergence," IEEE Trans. Antennas Propagat., vol. 59, no. 1, pp. 214-224, Jan. 2011.

[30] S. M. Hashemi, V. Nayyeri, M. Soleimani, and A.-R. Mallahzadeh, "Designing a compact-optimized planar dipole array antenna," IEEE Antennas Wirel. Propagat. Lett., vol. 10, pp. 243-246, 2011.

[31] K. V. Deligkaris, Z. D. Zaharis, D. G. Kampitaki, S. K. Goudos, I. T. Rekanos, and M. N. Spasos, "Thinned planar array design using Boolean PSO with velocity mutation", IEEE Trans. Magn., vol. 45, no. 3, pp. 1490-1493, March 2009.

[32] Z. D. Zaharis, "Radiation pattern shaping of a mobile base station antenna array using a particle swarm optimization based technique," Electr. Eng., vol. 90, no. 4, pp. 301-311, Apr. 2008.

[33] Z. D. Zaharis, D. G. Kampitaki, P. I. Lazaridis, A. I. Papastergiou, and P. B. Gallion, "On the design of multifrequency dividers suitable for GSM/DCS/PCS/UMTS applications by using a particle swarm optimization-based technique," Microw. Opt. Technol. Lett., vol. 49, no. 9, pp. 2138-2144, Sept. 2007.

[34] M. Fernandez Pantoja, A. R. Bretones, F. Garcia Ruiz, S. G. Garcia, and R. G. Martin, "Particle-Swarm optimization in antenna design: Optimization of log-periodic dipole arrays," IEEE Antennas Propagat. Mag., vol. 49, no. 4, pp. 34-47, Aug. 2007.

[35] Z. D. Zaharis, D. G. Kampitaki, P. I. Lazaridis, A. I. Papastergiou, A. T. Hatzigaidas, and P. B. Gallion, "Improving the radiation characteristics of a base station antenna array using a particle swarm optimizer," Microw. Opt. Technol. Lett., vol. 49, no. 7, pp. 1690-1698, July 2007.

[36] Z. D. Zaharis, I. P. Gravas, T. V. Yioultsis, P. I. Lazaridis, I. A. Glover, C. Skeberis, and T. D. Xenos, "Exponential log-periodic antenna design using improved particle swarm optimization with velocity mutation," IEEE Trans. Magn., vol. 53, no. 6, pp. 1-4, Article ID 7204104, June 2017.

[37] S. Goudos, "Antenna design using binary differential evolution: application to discrete-valued design problems," IEEE Antennas Propagat. Mag., vol. 59, no. 1, pp. 74-93, Feb. 2017.

[38] S. K. Goudos, K. A. Gotsis, K. Siakavara, E. E. Vafiadis, and J. N. Sahalos, "A multi-objective approach to subarrayed linear antenna arrays design based on memetic differential evolution," IEEE Trans. Antennas Propagat., vol. 61, no. 6, pp. 3042-3052, June 2013.

[39] Z. D. Zaharis, C. Skeberis, P. I. Lazaridis, and T. D. Xenos, "Optimal wideband LPDA design for efficient multimedia content delivery over emerging mobile computing systems," IEEE Syst. J., vol. 10, no. 2, pp. 831-838, Jun. 2016.

[40] S. Karimkashi, A. A. Kishk, and D. Kajfez, "Antenna array optimization using dipole models for MIMO applications," IEEE Trans. Antennas Propagat., vol. 59, no. 8, pp. 3112-3116, Aug. 2011. 
[41] S. Karimkashi and A. A. Kishk, "Invasive weed optimization and its features in electromagnetics," IEEE Trans. Antennas Propagat., vol. 58, no. 4, pp. 1269-1278, Apr. 2010.

[42] R. Cerf, "The quasispecies regime for the simple genetic algorithm with ranking selection," Trans. Am. Math. Soc., vol. 369, no. 9, pp. 60176071, 2017.

[43] CST Microwave Studio, CST AG, Germany.

[44] R. C. Hansen, Moment Methods in Antennas and Scattering. Norwood, MA: Artech House, 1990.

[45] G. J. Burke and A. J. Poggio, "Numerical Electromagnetics Code (NEC) - Method of Moments," Naval Ocean Systems Center, San Diego, CA, Tech. Doc. 116, Jan. 1981.

[46] A. Voors. (2015, Dec.). 4nec2. [Online]. Available: http://www.qsl.net/4nec2/

[47] B. B. Mangaraj, I. S. Misra, and S. K. Sanyal, "Application of bacteria foraging algorithm in designing log periodic dipole array for entire UHF TV spectrum," Int. J. RF Microw. Comput-Aid. Eng., vol. 23, no. 2, pp. 157-171, Mar. 2013.

[48] M. Touseef, Qamar-ud-Din, M. Aziz-ul-Haq, Umair Rafique, M. Arif Khan, and M. Mansoor Ahmed, "Genetic algorithm optimization of logperiodic dipole array," Int. J. Electromagnetics and Applications, vol. 2, no. 6, pp. 169-173, 2012.

[49] Xiao-Lin Zhang and Huo-Tao Gao, "An optimum design of miniaturized high frequency inverted-V log-periodic dipole antenna," in Proc. 2011 IEEE CIE Int. Conf. on Radar, Chengdu, China, 24-27 Oct. 2011, vol. 2, pp. 1185-1188.

[50] C. I. Tsitouri, S. C. Panagiotou, T. D. Dimousios, and C. N. Capsalis, "A circular switched parasitic array of log-periodic antennas with enhanced directivity and beam steering capability for ultra wideband communications applications," in Proc. 2008 Loughborough Antennas Propagat. Conf., Loughborough, UK, 17-18 Mar. 2008, pp. 281-284.

[51] T. L. Pitzer, A. James, G. B. Lamont, and A. J. Terzuoli, "Linear ensemble antennas resulting from the optimization of log periodic dipole arrays using genetic algorithms," in Proc. 2006 IEEE Congress on Evolutionary Computation, Vancouver, BC, Canada, 16-21 July 2006, pp. 3189-3196.

[52] S. E. El-Khamy, M. A. Mangoud, M. A. Aboul-Dahab, and A. I. Zaki, "Fractal multiband antennas using GA/MOM optimized log periodic dipole arrays," in Proc. 2004 IEEE Antennas Propagat. Society Int. Symp., Monterey, CA, USA, 20-25 June 2004, vol. 4, pp. 3433-3436.

[53] M. A. Mangoud, M. A. Aboul-Dahab, A. I. Zaki, and S. E. El-Khamy, "Genetic algorithm design of compressed log periodic dipole array," in Proc. 2003 IEEE 46th Midwest Symp. Circuits and Systems, Cairo, Egypt, 27-30 Dec. 2003, vol. 3, pp. 1194-1197.

[54] Y. C. Chung and R. Haupt, "Log-periodic dipole array optimization," $J$. Electromagn. Waves Appl., vol. 15, no. 9, pp. 1269-1280, 2001.

[55] S. Y. Stroobandt. (2017, Oct.). Parallel Square Conductor Transmission Line Calculator. [Online]. Available: http://www.stroobandt.com/zc.square/en/index.html

[56] O. Duffy. (2009, July). Characteristic impedance of transmission line of two square conductors in air. [Online]. Available: http://owenduffy.net/calc/tstl.htm

[57] D. Kirkby. (1996, Dec.). Finding the characteristics of arbitrary transmission lines. QEX. [Online]. pp. 3-10. Available: http://atlc.sourceforge.net/qex-december-1996/atlc.pdf

[58] IEEE Standard for Definitions of Terms for Antennas, IEEE Std 1452013. 